\title{
Ultrasonographic features of ductal carcinoma in situ: analysis of 219 lesions
}

\author{
Jun Kang Li ${ }^{1}$, Huan Fan Wang ${ }^{2}$, Yan He ${ }^{3}$, Yong Huang ${ }^{4}$, Gang Liu ${ }^{5}$, Zhi Li Wang ${ }^{3}$ \\ ${ }^{1}$ Department of Ultrasound, Chinese People's Liberation Army 63820 Hospital, Mianyang, China; ${ }^{2}$ Department of Ultrasound, Second Affiliated \\ Hospital of Xingtai Medical College, Xingtai, China; ${ }^{3}$ Department of Ultrasound, Chinese People's Liberation Army General Hospital, Beijing, \\ China; ${ }^{4}$ Department of Pathology, Chinese People's Liberation Army 63820 Hospital, Mianyang, China; ${ }^{5}$ Department of Radiology, Chinese People's \\ Liberation Army General Hospital, Beijing, China \\ Contributions: (I) Conception and design: JK Li, ZL Wang; (II) Administrative support: None; (III) Provision of study materials or patients: All \\ authors; (IV) Collection and assembly of data: All authors; (V) Data analysis and interpretation: All authors; (VI) Manuscript writing: All authors; (VII) \\ Final approval of manuscript: All authors. \\ Correspondence to: Zhi Li Wang, MD; Gang Liu, MD. Chinese People's Liberation Army General Hospital, 28 Fuxing Road, Beijing, 100853, China. \\ Email: wzllg@sina.com; lgwzl@126.com.
}

Background The purpose of this paper is to clarify the ultrasonographic features and classification of ductal carcinoma in situ (DCIS), and to evaluate the ability of ultrasonography in the prediction of DCIS.

Methods: The clinical data, gray-scale ultrasound images and pathological results of 219 DCIS lesions that detected in 203 consecutive patients who underwent ultrasonography and surgery in our hospital from January 1, 2014 to December 31, 2019 were collected retrospectively. Ultrasonographic features and classification of DCIS were summarized, and the accuracy of ultrasonography in predicting different ultrasonographic findings of DCIS were compared.

Results: Among the 219 DCIS lesions, 91 (41.6\%) presented as mass-like lesions and 128 (58.4\%) were non-mass-like lesions. For the 91 mass-like DCIS lesions, 79 were hypoechoic solid masses, 12 were cysticsolid structures. For the 128 non-mass-like DCIS lesions, 114 were hypoechoic areas, 10 were ductal dilatation accompanied with intraductal solid components, and 4 were multiple punctate echogenic foci only. The diagnostic accuracy of ultrasound for the 219 DCIS lesions was $81.7 \%(179 / 219)$. The diagnostic accuracy of mass-like DCIS lesions was $90.1 \%$ (82/91), which was significantly higher than that in nonmass-like DCIS lesions [75.8\% (97/128), P=0.007]. The diagnostic accuracy of hypoechoic solid masses was significantly higher than those of the other ultrasonographic findings $(\mathrm{P}=0.002)$. Ducts abnormalities were detected in 45 (20.5\%) lesions and punctate echogenic foci in $134(61.2 \%)$ lesions. The diagnostic accuracy of lesions with ducts abnormalities was $93.3 \%$ (42/45), which was significantly higher than that in lesions without ducts abnormalities [78.7\% (137/174), $\mathrm{P}=0.024]$. The diagnostic accuracy of lesions with punctate echogenic foci was $92.5 \%(124 / 134)$, which was significantly higher than that in lesions without punctate echogenic foci $[64.7 \%(55 / 85), \mathrm{P}=0.000]$.

Conclusions: DCIS lesions can effectively be recognized as mass-like lesions and non-mass-like lesions by ultrasound. Hypoechoic areas and hypoechoic solid masses were the most common ultrasonographic features of DCIS. Ducts abnormalities and punctate echogenic foci were helpful for the diagnosis of DCIS.

Keywords: Ductal carcinoma in situ (DCIS); duct abnormality; echogenic foci; ultrasonography

Submitted Mar 31, 2020. Accepted for publication Sep 18, 2020.

doi: $10.21037 / \mathrm{gs}-20-428$

View this article at: http://dx.doi.org/10.21037/gs-20-428 


\section{Introduction}

Ductal carcinoma in situ (DCIS) is a kind of non-invasive breast cancer characterized by malignant proliferation of breast ductal epithelial cells confined to the mammary ductal system without breaking through the basement membrane into surrounding stroma (1). The risk factors of DCIS may be related to the family history of breast cancer, age, the number of years from menarche to the first live birth, the frequency of delivery and the status of menopause. These risk factors were similar to those for invasive breast cancer $(2,3)$. Although DCIS is a non-fatal cancer in the early stage, and many patients with low-grade DCIS even can survive for more than forty years, but about 14-53\% may develop into invasive cancer given sufficient time. Of the untreated DCIS patients, the risk of developing invasive breast cancer in ipsilateral breast was significantly increased $(4,5)$. Therefore, early diagnosis and treatment are the most important measures to prevent DCIS from developing into invasive breast cancer $(6,7)$. Management strategies for DCIS treatment include surgery (mastectomy or lumpectomy), radiation therapy, and adjuvant endocrine therapy. The National Comprehensive Cancer Network Clinical Practice Guidelines in Oncology for Breast Cancer (8) recommend that primary treatment options for DCIS are lumpectomy plus whole breast radiation therapy with or without boost to tumor bed, total mastectomy with or without sentinel node biopsy, and lumpectomy alone. And then, endocrine therapy with tamoxifen or an aromatase inhibitor may be considered as a strategy to reduce the risk of recurrence in women with estrogen receptor positive DCIS.

The manners for the detection of DCIS were mainly mammography (MG), ultrasound, and magnetic resonance imaging (MRI). MRI has been prospectively shown to have a sensitivity of up to $98 \%$ for high-grade DCIS by using new techniques and sequences to show ductal tree and evaluation intraductal lesions. However, the high price, time-consuming and complex operation make it unable to be widely used $(9,10)$. MG is routinely used for the screening of breast cancer because it can show microcalcification sensitively. Previous studies have reported that MG has a sensitivity of up to $88 \%$ for DCIS (11). As a result of the widely use of MG, the detection rate of DCIS keeps growing, approximately 58,490 new cases of DCIS were diagnosed in the United States in 2015 (approximately 211,240 new breast cancer cases among women) $(12,13)$. However, MG may lead to false negative results, especially for patients with dense breast tissue, lesions without microcalcification, and tiny lesions.

Ultrasound has many advantages such as easy to use, real-time image, more efficient in examination of the dense breast tissue, and the ability to detect small lesions and focal dilated breast ducts negative on MG, which make the application of ultrasound in breast cancer screening more and more popular. Especially in the "two cancers" (cervical cancer and breast cancer) screening project in China, ultrasound plays an important role. In China, almost all hospitals and private physical examination institutions were equipped with high-performance ultrasound equipment, and a large number of DCIS cases were detected every year. It is well known that microcalcification is one of the most typical features of DCIS on MG, but little is known about its features on ultrasound. Previous studies have reported that DCIS presented as an irregular hypoechoic mass with an indistinct margin, microcalcifications, ductal changes, and structural distortion, but few reports focused on systematic analysis of ultrasonographic features and ducts abnormalities of DCIS $(14,15)$. The ultrasonographic features of DCIS are not specific, and there is variability in interpretation, understanding the classification of the features can improve the diagnostic accuracy. The evaluation of ducts abnormalities will provide valuable clues to predict the benign or malignant of breast lesions. The evaluation of the ability of ultrasonography in predicting DCIS can provide useful message for clinical decision. In this study, the ultrasonographic features and classification of DCIS were summarized, and the ability of ultrasonography in the prediction of DCIS was evaluated.

We present the following article in accordance with the STARD reporting checklist (available at http://dx.doi. org/10.21037/gs-20-428).

\section{Methods}

\section{Patients}

The study was conducted in accordance with the Declaration of Helsinki (as revised in 2013). The study was approved by the Medical Ethics Committee of Chinese People's Liberation Army General Hospital (NO.: S2020354-01) and individual consent for this retrospective analysis was waived. In this respective study, the database of patients was searched to identify all patients who were examined by ultrasound and underwent surgery in Chinese People's Liberation Army General Hospital from January 
1, 2014 to December 31, 2019. With histopathological results of the surgical specimens as the diagnostic standard (histopathological results were DCIS, without invasive component and lymph node metastasis), patients with invasive cancer or only biopsy were performed without surgery were excluded, 206 consecutive patients of DCIS were identified. Three patients were excluded again because they had received radiotherapy or endocrine therapy before ultrasonography, and 203 patients with 219 lesions were enrolled. The information of clinical data, gray-scale ultrasound images and pathological results were collected retrospectively.

\section{Ultrasound}

All patients underwent ultrasonography by sonographers with more than 5 years of experience in ultrasound of breast. Gray-scale ultrasonography were performed using high-frequency linear-array transducers: Philips IU22 with a L12-5 linear array probe, Philips EPIQ7 with a L125 linear array probe, Mindray Resona7 with a L11-3U linear array probe. The real-time images and static images were normalized stored in the database of our hospital, and the size was recorded in the reports (including length, width and height). In this study, the size was defined as the maximum length or maximum diameter for masses or areas, and ducts abnormalities were defined as the distortion or dilatation of surrounding branch ducts of hypoechoic areas, ductal extension, ductal dilatation alone, whether accompanied with internal echoes or not. All terms were described according to the American College of Radiology Breast Imaging Reporting and Data System (ACR BIRADS) (16). BI-RADS categories 1-4a were taken as benign and the BI-RADS scores of $4 \mathrm{~b}$ to 5 were taken as malignant. Shapes were classified as oval, round, and irregular; orientations were classified as parallel and nonparallel; margins were classified as well-defined, microlobulated, angular, indistinct, and spiculated. Echogenicity of the lesion was defined according to the echo of fat and normal breast tissue: "hypoechoic" means that the echo is lower than fat. "Isoechoic" means that the echo is similar to fat; "hyperechoic" means that the echo is similar to normal breast tissue or higher than the fat.

All images were analyzed by two doctors (ZLW and $\mathrm{YH})$ with more than 10 years of experience in ultrasound of breast without knowing the pathological results. Final decisions were reached by consensus. It is important to note that a patient's ultrasound image is not clear enough to be effectively assessed, and the diagnosis is based on the report previously stored.

\section{Statistical analyses}

Statistical analyses were performed with SPSS 25.0 (SPSS Inc., Chicago, IL, USA). Continuous data was described as mean \pm standard deviation (SD). Statistical comparisons were performed using the $\chi^{2}$ test or Fisher's exact test for categorical variables and Mann-Whitney $U$ test for mean size and mean age. Differences were considered to be statistically significant at $\mathrm{P}$ values $<0.05$.

\section{Results}

\section{Epidemiological characteristics}

All the 203 patients were women, age 26-87 years, the mean age was $49.7 \pm 11.6$ years (mean \pm SD), 110 patients had palpable masses, 7 patients had nipple discharge, and 86 patients had no obvious symptoms. No adverse events were reported in all patients undergoing ultrasound examinations.

\section{Ultrasonographic features and diagnostic accuracy}

The diagnostic accuracy of ultrasound for the 219 DCIS lesions was $81.7 \%$. Among the 219 DCIS lesions, 91 (41.6\%) presented as mass-like lesions and 128 (58.4\%) were non-mass-like lesions. For the 91 mass-like DCIS lesions, 79 were hypoechoic solid masses (Figure 1), 12 were cystic-solid structures (Figure 2). For the 128 nonmass-like DCIS lesions, 114 were hypoechoic areas (Figure 3), 10 were ductal dilatation accompanied with intraductal solid components (Figure 4), and 4 were multiple punctate echogenic foci only (Figure 5). Hypoechoic areas and hypoechoic solid masses were the most common ultrasonographic findings of DCIS, and 193 of 219 (88.1\%) lesions presented as hypoechoic areas or hypoechoic solid masses. Ultrasonographic findings, BI-RADS categories and diagnostic accuracy of 219 DCIS lesions are showed in Table 1.

The diagnostic accuracy of hypoechoic solid masses was significantly higher than those of the other ultrasonographic findings $(\mathrm{P}=0.002)$.

The differences between the mass-like DCIS lesions and the non-mass-like DCIS lesions are showed in Table 2. Significant differences of the mean size, punctate echogenic foci and diagnostic accuracy were found between mass-like 


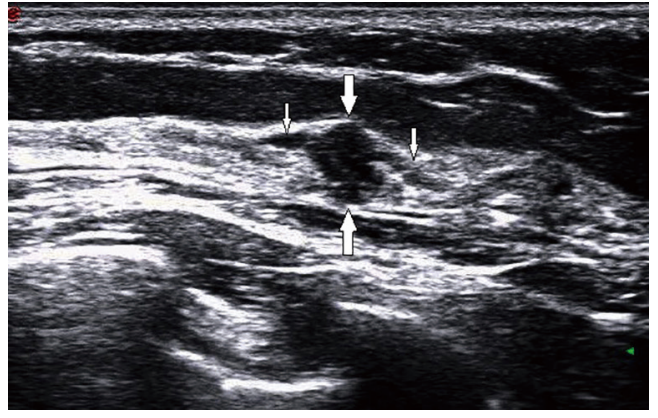

Figure 1 An unpalpable mass in the left breast of a 41-year-old woman. Ultrasound image showed an oval hypoechoic solid mass (large arrows) with well-defined margin, nonparallel orientation, ductal extension across the mass (small arrows). Histopathology revealed ductal carcinoma in situ.

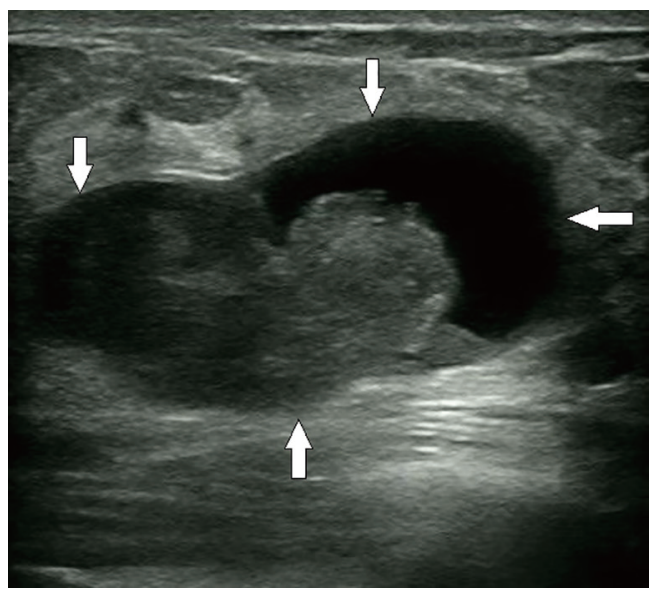

Figure 2 A palpable mass in the left breast of a 42 -year-old woman. Ultrasound image showed a cystic-solid structure (arrows) with well-defined margin, parallel orientation, solid components with an irregular shape. Histopathology revealed ductal carcinoma in situ.

DCIS lesions and non-mass-like DCIS lesions $(\mathrm{P}=0.000$, $\mathrm{P}=0.000, \mathrm{P}=0.007)$.

\section{Associated features}

Among the 219 DCIS lesions, 45 (20.5\%) were accompanied with ducts abnormalities. Ducts abnormalities were divided into four types: the distortion or dilatation of surrounding branch ducts of hypoechoic areas, ductal extension with hypoechoic areas, ductal extension with hypoechoic solid masses (across the lesions, connected to

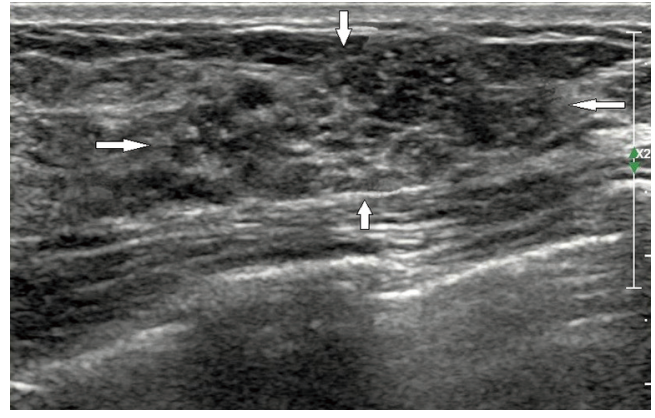

Figure 3 A palpable mass in the left breast of a 39-year-old woman. Ultrasound image showed an irregular hypoechoic area (arrows) with indistinct margin, parallel orientation, accompanied with internal punctate echogenic foci. Histopathology revealed ductal carcinoma in situ.

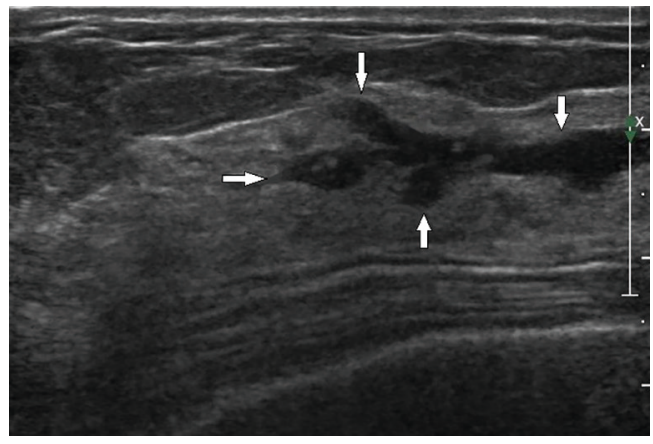

Figure 4 An unpalpable mass in the right breast of a 42 -year-old woman. Ultrasound image showed a ductal dilatation accompanied with intraductal solid components (arrows), branching shape, accompanied with internal punctate echogenic foci. Histopathology revealed ductal carcinoma in situ.

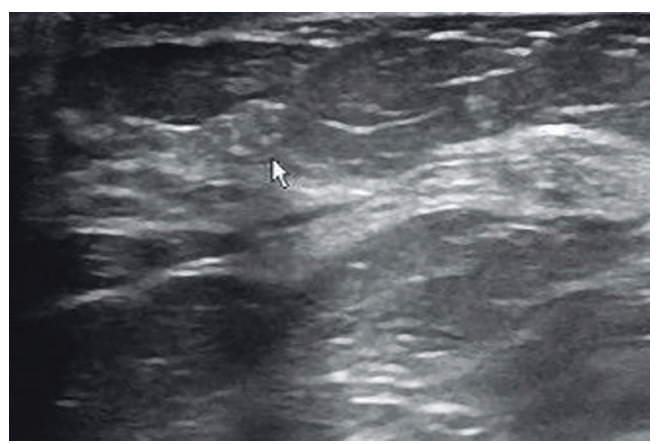

Figure 5 An unpalpable mass in the left breast of a 63-year-old woman. Ultrasound image showed multiple punctate echogenic foci only (arrow). Histopathology revealed ductal carcinoma in situ. 
Table 1 Ultrasonographic findings, BI-RADS categories and diagnostic accuracy of 219 DCIS lesions

\begin{tabular}{|c|c|c|c|c|c|c|c|}
\hline Ultrasonographic findings & \multicolumn{5}{|c|}{ BI-RADS categories } & Total, n (\%) & Accuracy, n (\%) \\
\hline \multicolumn{8}{|l|}{ Mass-like } \\
\hline Hypoechoic solid masses & 2 & 4 & 47 & 13 & 13 & $79(36.1)$ & $73(92.4)^{*}$ \\
\hline Cystic-solid structure & 1 & 2 & 5 & 1 & 3 & $12(5.5)$ & $9(75.0)$ \\
\hline Hypoechoic areas & 4 & 24 & 34 & 34 & 18 & $114(52.1)$ & $86(75.4)$ \\
\hline $\begin{array}{l}\text { Ductal dilatation accompanied with intraductal } \\
\text { solid components }\end{array}$ & 1 & 1 & 4 & 3 & 1 & $10(4.6)$ & $8(80.0)$ \\
\hline Multiple punctate echogenic foci only & 0 & 1 & 3 & 0 & 0 & $4(1.8)$ & $3(75.0)$ \\
\hline Variables & $e(n=91)$ & & & -mas & $e(n=128)$ & & $P$ value \\
\hline Mean size $(\mathrm{cm})$ & 0.8 & & & & & & $0.000^{*}$ \\
\hline Mean age (years) & 10.5 & & & & & & 0.110 \\
\hline With punctate echogenic foci & & & & & & & $0.000^{*}$ \\
\hline Yes & 7.3) & & & & & & \\
\hline
\end{tabular}

${ }^{*}$, implied statistical significance. DCIS, ductal carcinoma in situ.

lesions), only ductal dilatation accompanied with intraductal solid components (focal irregular dilatation of a single duct, branching shape). The classifications and diagrams of ducts abnormalities are showed in Table 3. The diagnostic accuracy of the lesions with ducts abnormalities was $93.3 \%$, which was significantly higher than that of without ducts abnormalities (93.3\% vs. $78.7 \%, \mathrm{P}=0.024)$.

There were 134 (61.2\%) lesions accompanied with punctate echogenic foci. The diagnostic accuracy of the lesions with punctate echogenic foci was $92.5 \%$, which was significantly higher than those of without punctate echogenic foci $(92.5 \%$ vs. $64.7 \%, \mathrm{P}=0.000)$.

\section{Discussion}

With the development of ultrasound equipment, ultrasound can be effectively used in the diagnosis of benign and malignant breast diseases. Up to now, ultrasound has become an important complementary tool in breast cancer screening, especially in cases without microcalcification on MG or young people with dense breast tissue $(17,18)$. Therefore, understanding the ultrasonographic features of DCIS is of great significance to the diagnosis of DCIS.

In this study, the diagnostic accuracy of ultrasound for the 219 DCIS lesions was $81.7 \%$, which indicates that there were limitations of ultrasound in the diagnosis of DCIS. Factors such as the diversity of ultrasonographic images of DCIS lesions, the inherent limitations of ultrasound, and the experience of sonographers have brought some difficulties to the diagnosis of DCIS by ultrasound. When benign lesions and malignant lesions cannot be distinguished, biopsy is inevitable. In our series, lesions classified as BI-RADS 4 category were the most frequent (80.4\%), which confirmed that most DCIS lesions required 
Table 3 The classifications and diagrams of ducts abnormalities of 45 DCIS lesions

Ducts abnormalities
Distortion or dilatation of surrounding branch ducts of hypoechoic
areas
Ductal extension with hypoechoic areas
Ductal extension with hypoechoic solid masses
Connected to lesion
Across the lesion
Only ductal dilatation accompanied with intraductal solid
Focal irregular dilatation of a single duct
Branching shape

DCIS, ductal carcinoma in situ.

further examination, mainly biopsy.

In this study, DCIS lesions presented as mass-like lesions or non-mass-like lesions in ultrasonographic images. Previous studies had focused on the non-mass-like lesions of DCIS (11,19-21). Uematsu et al. (21) defined nonmass-like lesions as an area that had different echogenicity from adjacent breast tissues or the same area in the contralateral breast, accompanied with indistinct margins and undefined shapes, and without space-occupying effect in two different projections. The proportion of non-masslike lesions of DCIS was reported to range from $25 \%$ to $60 \%$. In this study, $58.4 \%$ of DCIS lesions were presented as non-mass-like lesions. It is reported that the processes of the development of DCIS are complex and diverse $(5,22)$. Franks et al. (22) developed a mathematical model to model the initial stages of the growth of DCIS. Under the mathematical model, the authors suggested that the interaction between the viscosity of malignant cells and nutrient concentrations were fundamental elements of the expansion of malignant cells. Malignant cells proliferate continuously after it developed primarily at terminal duct lobular unit (TDLU). When malignant cells proliferate in a localized area and do not spread in ductal system, the image showed as mass-like lesions. When malignant cells mainly spread into the central or branch ducts and these ducts thereby distortion or dilatation, the image showed as ducts abnormalities. When malignant cells were spread widely within branch ducts and interaction between the intraductal component and the stroma, the images showed as hypoechoic areas $(19,23)$. Different types of proliferation of malignant cells can developed simultaneously or successively, and then a variety of combinations of these types showed various ultrasonographic features.

Hypoechoic areas and hypoechoic solid masses were the most common ultrasonographic findings, followed by cystic-solid structure and ductal dilatation accompanied with intraductal solid components, multiple punctate echogenic foci only were rare. Watanabe et al. (19) classified the ultrasound findings of DCIS into solid or mixed masses, ducts abnormalities, hypoechoic areas in the mammary gland, architectural distortion, multiple small cysts, echogenic foci without a hypoechoic area. There was difference between these two studies in the category of DCIS, which might be caused by different recognition of non-mass-like lesions. Architectural distortion and multiple small cysts were classified into hypoechoic areas in this study, and the architectural distortion was difficult to be identified in static images.

The mean size of the non-mass-like DCIS lesions were significantly larger than mass-like DCIS lesions and nonmass-like DCIS lesions were more frequently associated with punctate echogenic foci. The reason for the difference of punctate echogenic foci might be that non-mass-like DCIS lesions on ultrasound were mainly associated with high-grade DCIS, and punctate echogenic foci were more frequently detected in high-grade lesions than lowgrade lesions and in comedo lesions than in noncomedo lesions $(24,25)$. Due to the different development processes 
mentioned above, the mean size of non-mass-like DCIS lesions was significantly larger than that of mass-like DICS lesions. The mean size of the non-mass-like DCIS lesions measured in this study might be smaller than the actual size, and the actual size of non-mass-like DCIS lesions is difficult to measure accurately, even in histopathological specimens (26).

In this study, the diagnostic accuracy of mass-like DCIS lesions was significantly higher than that in non-mass-like DCIS lesions. The reason for the difference might be that diagnostic criteria for non-mass-like lesions have not yet been established, standardized analysis of non-mass-like lesions cannot be performed, and the diagnosis of non-masslike lesions is susceptible to the subjectivity of sonographers. In fact, not only DCIS lesions, but also other malignant lesions and benign lesions can present as non-masslike lesions, such as infiltrating ductal carcinoma (IDC), lymphatic metastatic poorly differentiated adenocarcinoma, adenocarcinoma, acute lymphatic leukaemia, infiltrating lobular carcinoma (ILC), adenosis, intraductal papilloma and plasma cell mastitis $(21,27)$. According to the results of previous studies, mottled and geographic patterns, architectural distortion, posterior acoustic shadowing, microcalcifications, older (postmenopausal) patient age, and a larger lesion size were more frequently associated with malignant non-mass-like lesions than benign non-masslike lesions (28-32). Study of Wang et al. (33) observed that the elastic modulus, elastic modulus ratio and stiff rim sign of the malignant lesions were higher than those of benign, and the combination of conventional ultrasound and elastography could increase the diagnostic accuracy of non-mass-like breast lesions. For the 128 cases of nonmass-like DCIS lesions in this study, 26 (20.3\%) cases were diagnosed as BI-RADS 4a categories, even 5 (3.9\%) cases were misdiagnosed as BI-RADS 3 categories. Therefore, for the management of non-mass-like lesions diagnosed as BIRADS 4a categories, biopsy is warranted.

The diagnostic accuracy of hypoechoic solid masses was significantly higher than those of the other ultrasonographic findings. The BI-RADS sonographic lexicon was helpful in distinguishing benign from malignant hypoechoic solid masses with suspicious features of malignancy being irregular shape, nonparallel orientation, microlobulated or indistinct margin, etc.

Ducts abnormalities can improve the accuracy of ultrasound in predicting DCIS. In this study, $20.5 \%$ of DCIS lesions were accompanied with ducts abnormalities. A comparison of the diagnostic accuracy of the lesions with and without ducts abnormalities showed that the diagnostic accuracy of lesions with ducts abnormalities was significantly higher than the lesions without ducts abnormalities. The distortion or dilatation of surrounding branch ducts of hypoechoic areas and the branching shape of ductal dilatation accompanied with intraductal solid components might be due to malignant cells spreading along the ductal system and invading the branching ducts (19). Ductal extension might be due to malignant cells spreading along the ductal system and obstructing the peripheral ductal system (34). Although ducts abnormalities were important signs of DCIS, but not all lesions accompanied with ducts abnormalities were malignant, especially the lesions presented as focal irregular dilatation of a single duct with intraductal solid components. According to the data of previous studies, intraductal papillomas, ductal hyperplasia, fibrocystic changes, and fibrotic lesions also showed as ducts abnormalities on ultrasonographic images, and approximately $8-16 \%$ of intraductal masses were malignant $(34,35)$. Study of Kim et al. (35) demonstrated that symptoms, personal history of breast cancer, larger size, tended to fill the duct or extend outside the duct, involved branch duct were more frequently associated with malignant. Study of Hsu et al. (34) demonstrated that non-subareolar location, indistinct margins, and ducts adjacent to or across a mass increases the likelihood of malignancy. Intraductal solid components accompanied by multiple punctate echogenic foci were highly indicative of malignancy as well (36). All the breast ducts associated with hypoechoic areas, hypoechoic solid masses, and intraductal solid components should undergo biopsy, but this proposition needs to be validated with more data in the future.

Punctate echogenic foci were helpful for the diagnosis of DCIS. In this study, $61.2 \%$ of DCIS lesions were accompanied with punctate echogenic foci. The diagnostic accuracy of the lesions with punctate echogenic foci was significantly higher than the lesions without punctate echogenic foci. Punctate echogenic foci presented as segmental distribution, located in ducts, varied in size and shape, clusters larger than $10 \mathrm{~mm}$ were more frequently associated with malignancy (37-40). For the reason that punctate echogenic foci are tiny, without comet-tail artifact and posterior acoustic shadowing, it is easy to be missed during ultrasonography, especially for the lesions presented as multiple punctate echogenic foci only. The manners of adjustment of ultrasound settings, optimal probe pressure, review clinicopathological data are helpful for decrease the rate of false-negative findings (41). 
There are some limitations in our study. First, this study was a single center study with a relatively small number of cases, which may not reflect the epidemiological characteristics of DCIS. DCIS is an uncommon breast disease, data in a single center is general limited, and the true incidence may be higher than that in the real world. Nevertheless, this study is important because it reflects the epidemiological characteristics of DCIS in local areas, which may provide reference for future studies; The ultrasonographic features of DCIS summarized in this paper may provide useful clues for the diagnosis and treatment of breast diseases. Second, only the patients both underwent ultrasonography and histological results of the surgical specimen were DCIS were included in this study, which introduced an element of selection bias. Third, only gray-scale ultrasound images were evaluated in this study, while color doppler ultrasound, elastography and contrastenhanced ultrasound were not observed and evaluated. The prospective study by color doppler ultrasound, elastography and contrast-enhanced ultrasound will be added in the future.

\section{Conclusions}

DCIS lesions can effectively be recognized as masslike lesions and non-mass-like lesions by ultrasound. Hypoechoic areas and hypoechoic solid masses were the most common ultrasonographic features of DCIS. Ducts abnormalities and punctate echogenic foci were helpful for the diagnosis of DCIS.

\section{Acknowledgments}

Funding: This work was supported by the National Natural Science Foundation (grant number: 81771832), and Military Top project of Youth Training for Medical Science and Technology (grant number: 19QNP071).

\section{Footnote}

Reporting Checklist: The authors have completed the STARD reporting checklist. Available at http://dx.doi.org/10.21037/ gs-20-428

Data Sharing Statement: Available at http://dx.doi. org/10.21037/gs-20-428
Conflicts of Interest: All authors have completed the ICMJE uniform disclosure form (available at http://dx.doi. org/10.21037/gs-20-428). ZLW serves as an unpaid editorial board member of Gland Surgery from April, 2019 to March, 2021. The other authors have no conflicts of interest to declare.

Ethical Statement: The authors are accountable for all aspects of the work in ensuring that questions related to the accuracy or integrity of any part of the work are appropriately investigated and resolved. The study was conducted in accordance with the Declaration of Helsinki (as revised in 2013). The study was approved by the Medical Ethics Committee of Chinese People's Liberation Army General Hospital (NO.: S2020-354-01) and individual consent for this retrospective analysis was waived.

Open Access Statement: This is an Open Access article distributed in accordance with the Creative Commons Attribution-NonCommercial-NoDerivs 4.0 International License (CC BY-NC-ND 4.0), which permits the noncommercial replication and distribution of the article with the strict proviso that no changes or edits are made and the original work is properly cited (including links to both the formal publication through the relevant DOI and the license). See: https://creativecommons.org/licenses/by-nc-nd/4.0/.

\section{References}

1. Schnitt SJ, Silen W, Sadowsky NL, et al. Ductal carcinoma in situ (intraductal carcinoma) of the breast. $\mathrm{N}$ Engl J Med 1988;318:898-903.

2. Al-Ajmi K, Lophatananon A, Ollier W, et al. Risk of breast cancer in the UK biobank female cohort and its relationship to anthropometric and reproductive factors. PLoS One 2018;13:e0201097.

3. Peila R, Arthur R, Rohan TE. Risk factors for ductal carcinoma in situ of the breast in the UK Biobank cohort study. Cancer Epidemiol 2020;64:101648.

4. Collins LC, Tamimi RM, Baer HJ, et al. Outcome of patients with ductal carcinoma in situ untreated after diagnostic biopsy: results from the Nurses' Health Study. Cancer 2005;103:1778-84.

5. Erbas B, Provenzano E, Armes J, et al. The natural history of ductal carcinoma in situ of the breast: a review. Breast Cancer Res Treat 2006;97:135-44. 
6. Boughey JC, Gonzalez RJ, Bonner E, et al. Current treatment and clinical trial developments for ductal carcinoma in situ of the breast. Oncologist 2007;12:1276-87.

7. Bonnett M, Wallis T, Rossmann M, et al. Histologic and radiographic analysis of ductal carcinoma in situ diagnosed using stereotactic incisional core breast biopsy. Mod Pathol 2002;15:95-101.

8. Gradishar WJ, Anderson BO, Balassanian R, et al. Breast Cancer, Version 4.2017, NCCN Clinical Practice Guidelines in Oncology. J Natl Compr Canc Netw 2018;16:310-20.

9. Yilmaz R, Bender O, Celik Yabul F, et al. Diagnosis of Nipple Discharge: Value of Magnetic Resonance Imaging and Ultrasonography in Comparison with Ductoscopy. Balkan Med J 2017;34:119-26.

10. Kuhl CK, Schrading S, Bieling HB, et al. MRI for diagnosis of pure ductal carcinoma in situ: a prospective observational study. Lancet 2007;370:485-92.

11. Jin ZQ, Lin MY, Hao WQ, et al. Diagnostic evaluation of ductal carcinoma in situ of the breast: ultrasonographic, mammographic and histopathologic correlations. Ultrasound Med Biol 2015;41:47-55.

12. Ernster VL, Barclay J. Increases in ductal carcinoma in situ (DCIS) of the breast in relation to mammography: a dilemma. J Natl Cancer Inst Monogr 1997;(22):151-6.

13. Jemal A, Murray T, Ward E, et al. Cancer statistics, 2005. CA Cancer J Clin 2005;55:10-30.

14. Cha H, Chang YW, Lee EJ, et al. Ultrasonographic features of pure ductal carcinoma in situ of the breast: correlations with pathologic features and biological markers. Ultrasonography 2018;37:307-14.

15. Izumori A, Takebe K, Sato A. Ultrasound findings and histological features of ductal carcinoma in situ detected by ultrasound examination alone. Breast Cancer 2010;17:136-41.

16. Mendelson EB, Böhm-Vélez M, Berg WA, et al. ACR BI-RADS Ultrasound. In: ACR BI-RADS Atlas. Breast Imaging Reporting and Data System. 5th Edition. American College of Radiology, 2013:35-75.

17. Buchberger W, Niehoff A, Obrist $P$, et al. Clinically and mammographically occult breast lesions: detection and classification with high-resolution sonography. Semin Ultrasound CT MR 2000;21:325-36.

18. Okello J, Kisembo H, Bugeza S, et al. Breast cancer detection using sonography in women with mammographically dense breasts. BMC Med Imaging 2014;14:41.
19. Watanabe T, Yamaguchi T, Tsunoda H, et al. Ultrasound Image Classification of Ductal Carcinoma In Situ (DCIS) of the Breast: Analysis of 705 DCIS Lesions. Ultrasound Med Biol 2017;43:918-25.

20. Gunawardena DS, Burrows S, Taylor DB. Non-mass versus mass-like ultrasound patterns in ductal carcinoma in situ: is there an association with high-risk histology? Clin Radiol 2020;75:140-7.

21. Uematsu T. Non-mass-like lesions on breast ultrasonography: a systematic review. Breast Cancer 2012;19:295-301.

22. Franks SJ, Byrne HM, King JR, et al. Modelling the early growth of ductal carcinoma in situ of the breast. J Math Biol 2003;47:424-52.

23. Morishima I, Ueno E, Tohno E, et al. Ultrasonic Diagnosis of Non-Mass Image-Forming Breast Cancer. In: Ueno E, Shiina T, Kubota M, et al. editors. Research and Development in Breast Ultrasound. Tokyo: Springer, 2005:127-34.

24. Kim HR, Jung HK. Histopathology findings of nonmass cancers on breast ultrasound. Acta Radiol Open 2018;7:2058460118774957.

25. Scoggins ME, Fox PS, Kuerer HM, et al. Correlation between sonographic findings and clinicopathologic and biologic features of pure ductal carcinoma in situ in 691 patients. AJR Am J Roentgenol 2015;204:878-88.

26. Dadmanesh F, Fan X, Dastane A, et al. Comparative analysis of size estimation by mapping and counting number of blocks with ductal carcinoma in situ in breast excision specimens. Arch Pathol Lab Med 2009;133:26-30.

27. Wang ZL, Li N, Li M, et al. Non-mass-like lesions on breast ultrasound: classification and correlation with histology. Radiol Med 2015;120:905-10.

28. Kim SJ, Park YM, Jung HK. Nonmasslike lesions on breast sonography: comparison between benign and malignant lesions. J Ultrasound Med 2014;33:421-30.

29. Li L, Zhou X, Zhao X, et al. B-Mode Ultrasound Combined with Color Doppler and Strain Elastography in the Diagnosis of Non-mass Breast Lesions: A Prospective Study. Ultrasound Med Biol 2017;43:2582-90.

30. Park JW, Ko KH, Kim EK, et al. Non-mass breast lesions on ultrasound: final outcomes and predictors of malignancy. Acta Radiol 2017;58:1054-60.

31. Qu XX, Song Y, Zhang YH, et al. Value of Ultrasonic Elastography and Conventional Ultrasonography in the Differential Diagnosis of Non-Mass-like Breast Lesions. Ultrasound Med Biol 2019;45:1358-66.

32. Newburg AR, Chhor CM, Young Lin LL, et al. Magnetic 
Resonance Imaging-Directed Ultrasound Imaging of Non-Mass Enhancement in the Breast: Outcomes and Frequency of Malignancy. J Ultrasound Med 2017;36:493-504.

33. Wang ZL, Li Y, Wan WB, et al. Shear-Wave Elastography: Could it be Helpful for the Diagnosis of Non-Mass-Like Breast Lesions? Ultrasound Med Biol 2017;43:83-90.

34. Hsu HH, Yu JC, Hsu GC, et al. Ultrasonographic alterations associated with the dilatation of mammary ducts: feature analysis and BI-RADS assessment. Eur Radiol 2010;20:293-302.

35. Kim WH, Chang JM, Moon WK, et al. Intraductal mass on breast ultrasound: final outcomes and predictors of malignancy. AJR Am J Roentgenol 2013;200:932-7.

36. Ban K, Tsunoda H, Watanabe T, et al. Characteristics of ultrasonographic images of ductal carcinoma in situ

Cite this article as: Li JK, Wang HF, He Y, Huang Y, Liu G, Wang ZL. Ultrasonographic features of ductal carcinoma in situ: analysis of 219 lesions. Gland Surg 2020;9(6):1945-1954. doi: $10.21037 / g s-20-428$ with abnormalities of the ducts. J Med Ultrason (2001) 2020;47:107-15.

37. Hashimoto BE. Sonographic assessment of breast calcifications. Curr Probl Diagn Radiol 2006;35:213-8.

38. Kang SS, Ko EY, Han BK, et al. Breast US in patients who had microcalcifications with low concern of malignancy on screening mammography. Eur J Radiol 2008;67:285-91.

39. Nagashima T, Hashimoto H, Oshida K, et al. Ultrasound Demonstration of Mammographically Detected Microcalcifications in Patients with Ductal Carcinoma in situ of the Breast. Breast Cancer 2005;12:216-20.

40. Moon WK, Im JG, Koh YH, et al. US of mammographically detected clustered microcalcifications. Radiology 2000;217:849-54.

41. Uematsu T. Ultrasonographic findings of missed breast cancer: pitfalls and pearls. Breast Cancer 2014;21:10-9. 